\title{
Multi-point monitoring of unstable slope with low cost sensor network
}

\author{
Taro Uchimura ${ }^{\text {i) }}$, Lin Wang ${ }^{\text {ii) }}$, Hiroshi Yamaguchi ${ }^{\text {ii)}}$, Shunsaku Nishie ii), Ippei Eto ${ }^{\text {i) }}$, \\ Shangning Tao ${ }^{\text {i)}}$, Chin-Wei Lu ${ }^{\text {iii)}}$, Jui-Jen Chang ${ }^{\text {iv) }}$, Chien-Wen Chen ${ }^{\text {iii) }}$ \\ i) University of Tokyo, 7-3-1 Hongo, Bunkyo City, Tokyo, Japan \\ ii) Chuo Kaihatsu Corporation, 3-13-5 Nishiwaseda, Shinjuku City, Tokyo, Japan \\ iii) National Kaohsiung First University of Science and Technology, Kaohsiung, Taiwan \\ iv) Geofield Engineering Consultants Inc., Kaohsiung, Taiwan
}

\begin{abstract}
A low-cost and simple monitoring method for early warning of rainfall-induced landslides has been proposed by the authors. Tilting angles in the surface layer of slope are mainly monitored in this method. A set of equipment has been developed for practical use, which is equipped with a MEMS (Micro Electro Mechanical Systems) tilt sensor and a volumetric water content sensor. In several case studies with this system, including a slope failure test conducted on a natural slope by applying artificial heavy rainfall, it detected distinct behaviors in the tilting angles in the pre-failure stages. Considering these behaviors, the authors has proposed to issue precaution at a tilting rate of 0.01 degrees per hour, and warning at that of 0.1 degree per hour, for conservative decision.

The development of this system reduced the cost for slope monitoring reasonably. However, the number of available equipment for each slope is still limited due to financial restriction, and therefore, engineers need to select the position of sensors carefully for effective early warning. This paper introduce authors' recent attempt to develop more low-cost sensor network for slope monitoring. The cost for each sensor node was reduced by around one-third, and consequently, slopes can be monitored at many point, resulting in more meticulous observation of slope behaviors.
\end{abstract}

Keywords: slope failure, early warning, monitoring, tilt sensors, wireless network

\section{INTRODUCTION}

There is a long history in prevention and mitigation of rainfall/scouring-induced landslides. Mechanical counter measures to prevent slope failure, like retaining walls and ground anchors, have been widely used. However, they are expensive and it is not realistic to apply such mechanical measures for all of these slopes with potential risk, because most of landslide occurs in small scale, but a large number of slopes. Therefore, careful monitoring of slope behaviors and consequent early warning is reasonable as alternatives.

The authors have proposed and developed an early warning system for slope failures, as one of feasible countermeasures (Figure 1) (Uchimura, et. al. 2010 \& 2011). The system consists of minimum number of lowcost sensors on a slope, and the data is transmitted through wireless network. Thus, the system is low-cost and simple so that the residents in risk areas can handle it to protect themselves from slope disasters.

Uchimura et. al. (2015) summarized case studies on the tilting-rates observed in slope surface in pre-failure stages obtained at 5 natural slope sites under natural or artificial heavy fainfall. In Figure 2, the vertical axis presents "the duration before failure or stabilization". That is, in cases the slope failed at the position of the tilt sensor, the duration is measured from the time when the corresponding tilting rate was observed to the time of failure. In cases the slope was did not fail and stabilized finally, the duration is measured from the time when the corresponding tilting rate was observed to the time of when the slope was stabilized.

According to Figure 2, the order of tilting rate observed with slope deformation varied widely from $10^{-}$ ${ }^{4}$ to 10 degree/hour depending on situations. The tilting rate tends to increase toward failure, and a shorter duration is remaining before failure when higher tilting rate is observed. The observed tilting rate was more than 0.01 degree/hour for all the cases in which the slope

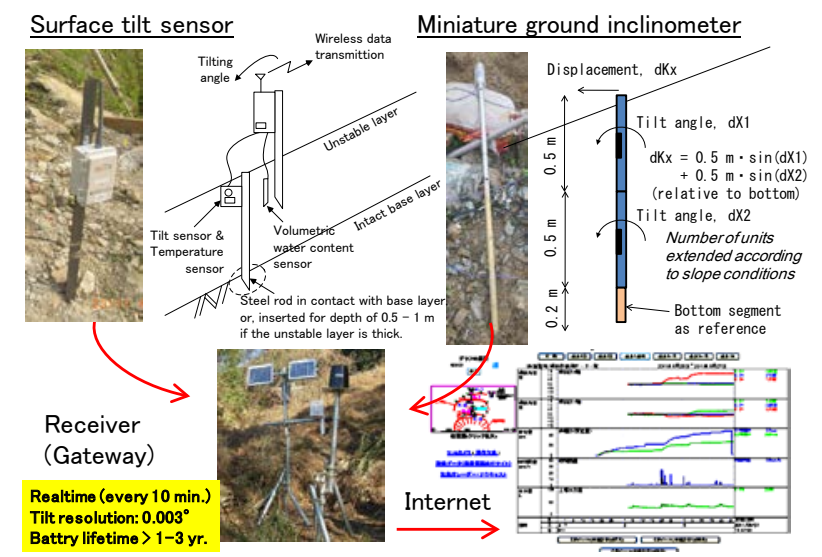

Figure 1. Tilt sensor units and wireless monitoring system 
failed or nearly failed finally, while it was less than 0.1 degree/hour for all other cases. Durations of 1 to 10 hours were remained before failure when at tilting rate of 0.1 degree/hour was observed.

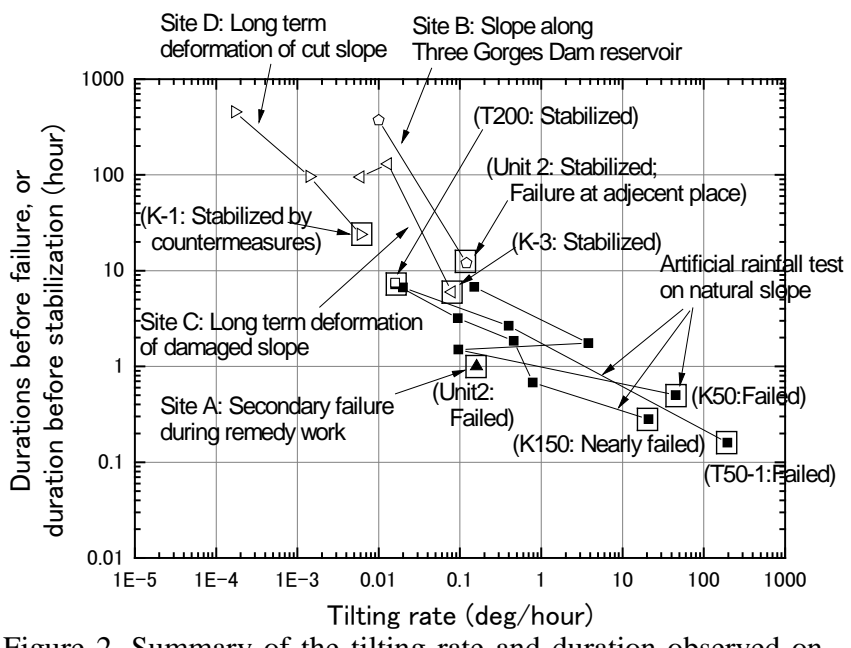

Figure 2. Summary of the tilting rate and duration observed on slope surface.

Based on these case studies, the authors came to an idea of issuing warning at a tilting rate exceeding 0.1 degree/hour, and precaution at that of 0.01 degrees/hour for safety. This paper makes further reference on the author's attempt to improve the applicability of the moniroting and early warning system. The sensor equipment is modified to be more low-cost, smaller in its size and weight, and simpler in instalation and operation. As a result, the total cost for the monitoring sytem is reduced, and larger number of sensors can be installed on the slope site. It helps us to figure out real-time situation of the slope in more details.

Figure 3 illustrates a typical arrangement of the equipment. The proposed new sensor units are more lowcost and simple than the conventional units, but they have relatively shorter distance of radio transmission (around $30 \mathrm{~m}$ under worst condition). They are arranged densely on the high-risk areas among the site, and one conventional unit collect all the data of each area. Then, the data is transmitted through longer distance (300 - 600 $\mathrm{m}$ ), and uploaded to the internet server. Thus, a meticulous sensor network can be constructed with reasonable cost.

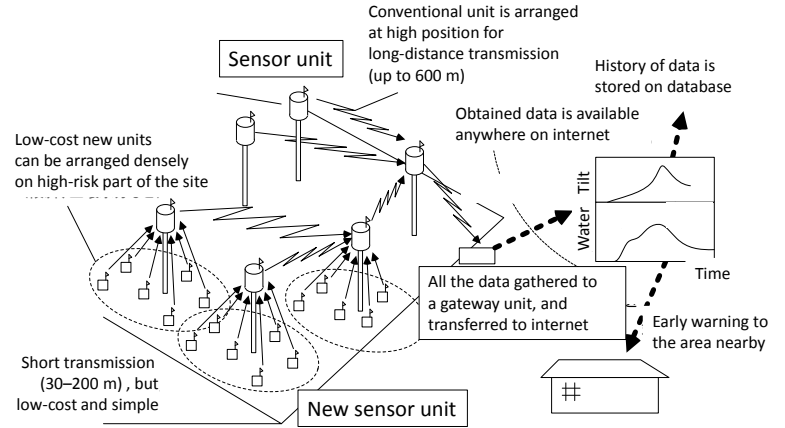

Figure 3. Typical arrangement of equipment including proposed new sensor units.

\section{DETAILED DESIGN OF NEW SENSOR UNIT}

\subsection{Basic specification}

Figure 4 shows appearance of the proposed new sensor unit. The controller/radio module is made smaller than the conventional one. A steel rod is inserted into the slope surface for a depth of $0.5-1 \mathrm{~m}$, and the tilt sensor module is attached on the rod at subground position to reduce data drift due to temperature changes. As the required transmission distance is relatively shorter, the unit can be placed at a lower position, $30-50 \mathrm{~cm}$ over the ground surface. It will reduce the size and weight of the sensor unit, resulting in lower cost and easy operation.

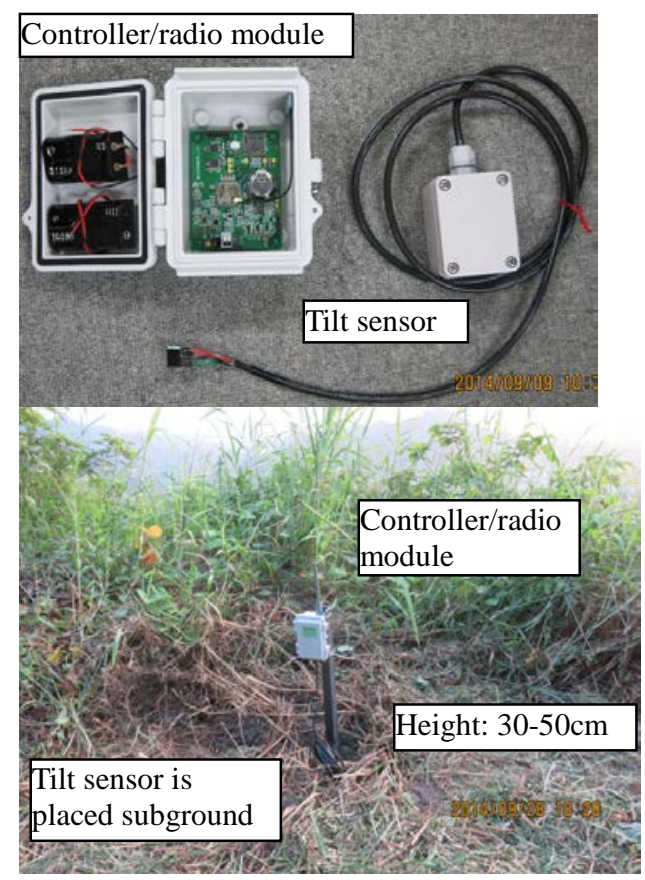

Figure 4. Appearance of the proposed new sensor unit.

In order to satisfy the requirements on cost, power consumption, radio transmission distance, accuracy and reliability, a CPU (Central Processing Unit) with radioon-chip is used. The CPU is called Wireless Engine, provided by Tokyo Cosmos Electric Co., Ltd. It is a 32bit RISC microprocessor, can run for 1 to $32 \mathrm{MHz}$ with 32Kbytes RAM, 160K bytes Flash Memory, 4K EEPROM. It includes a $2.4 \mathrm{GHz}$ radio module on chip. And a protocol software is provided as a library, implementing an IEEE802.15.4 standards-based wireless transceiver that transmits and receives data by 250Kbps over the air in the unlicensed $2.4 \mathrm{GHz}$ band.

Each sensor unit comprises a CPU, a tilt sensor, a Micro SD card, a Real time clock, a LED, and a Battery set. The tilt sensors is MEMS sensor with low current consumption (5mA), low temperature dependency $\left( \pm 0.014 \% /{ }^{\circ} \mathrm{C}\right)$, high resolution of $0.0035^{\circ}(10 \mathrm{~Hz} \mathrm{BW}$, analog output)) and low noise. It can measure inclination ranges $\pm 30^{\circ}$ in dual axis ( $\mathrm{X}$ and $\mathrm{Y}$ ). It shows nonnegligible drift with temperature changes. Its characteristics are carefully measured for respective sensor chips, and the measured data is corrected with 
temperature data. A Micro SD card is used to record the measured data. A configuration file, which instruct the controller with unique information such as identification number, source address, destination address and wake up timing. The behaviors of each sensor unit can be rearranged easily by rewriting the configuration file. An LED is used to indicate the current status of the sensor unit to ensure reliable functions of each unit. Two AA alkaline batteries are used for each sensor unit. It can drive the unit for more than half a year with typical measuring interval of 10 minutes.

\subsection{Evaluation of radio transmission distance}

The conventional sensor unit has sufficiently long radio transmission distance, $300 \mathrm{~m}$, or $600 \mathrm{~m}$ under good conditions. However, the proposed new sensor unit has shorter transmission distance, because it uses higher radio frequency ( $2.4 \mathrm{GHz}$ band) than the conventional one (430 $\mathrm{MHz}$ band). The available distance was carefully evaluated for reasonable arrangement of sensors (Figure 5). The receiver was fix at a height of 1 $\mathrm{m}$, while the transmitter was set at several heights and distances, and the radio signal recognition was checked.

Table 1 shows the results. On a flat plane (a wide open space with horizontal and paved ground surface), the signal reached through a distance of $130-238 \mathrm{~m}$ depending on the height of antenna. Even on a slope with open area without vegetation, the signal reached through $215 \mathrm{~m}$. However, when it is tested in a forest with dense vegetation, the transmission distance reduced to $33-36$ $\mathrm{m}$. The radio signal with higher frequency intends to propagate more straight, significantly affected by obstacles between the transmitter and receiver has significant effect.

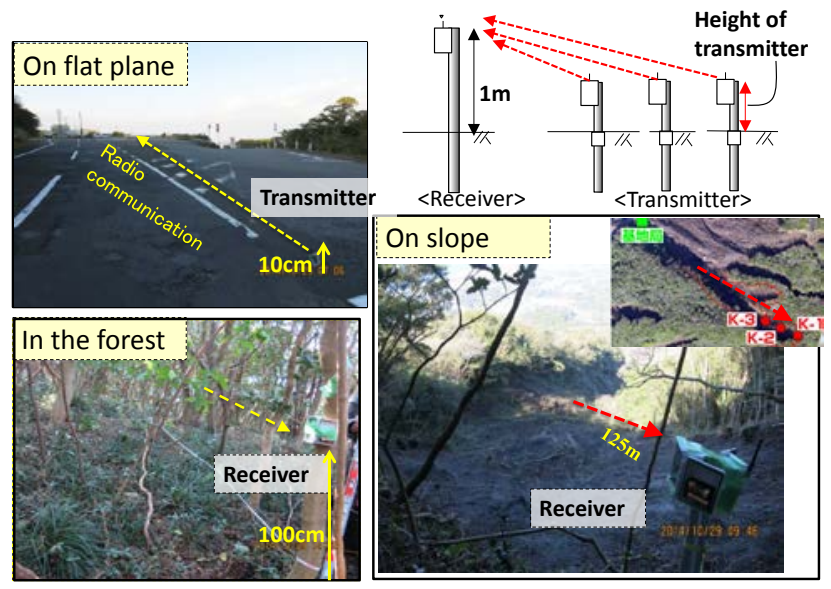

Figure 5. Evaluation of radio transmission distance.

Table 1. Summary of transmission distance under various conditions.

\begin{tabular}{|c|c|c|c|}
$\begin{array}{l}\text { Height of } \\
\text { transmitter }(\mathrm{cm})\end{array}$ & Flat Plane $(\mathrm{m})$ & Slope $(\mathrm{m})$ & Forest $(\mathrm{m})$ \\
\hline 10 & 130 & 215 & 33 \\
\hline 50 & 204 & - & 33 \\
\hline 100 & 238 & - & $34 \sim 36$ \\
\hline
\end{tabular}

\section{TRIAL INSTALLATION ON A CUT SLOPE}

The developed prototype of the proposed new sensor units were installed on a cut slope site in Yenchao, Kaohsiung City, Taiwan at the end of 2014. This is a municipal waste disposal site, and its cut slope started to move after excavation works for construction (Figure 6). There are two hazards occurred on this slope, one is a very shallow movement of the ground surface and the other is a deeper movement of the slope. Muddy soils spreads around on the passage at the slope after rains and slope deforms at toes. Although engineers have conducted hanging-net for vegetation slope protection, the soils around protection net were eroded. For a deeper slope stability protection, there is no special treatment at this moment, because not too many but one temple and one residential house locate nearby.

The ground dips to the toe, consisted of mainly mudstone at the depth 7-10m below the ground surface, and weathered soils and reclaimed soils at the shallower depth. The mudstone is a typical geotechnical material in this region, and its one dimensional compression strength is around $200-300 \mathrm{kPa}$ with an axial strain $6 \%$ (Figure 7). Some materials easily collapse when being immersed in water. The surface materials consisted of great amount of silt with liquid limit around 20.

Figure 8 indicates the arrangement of tilt sensors. Eighteen tilt sensors are installed to cover the moving block. The tilt angle data is measured every 10 minutes, and corrected by a receiver on the slope in the opposite side.

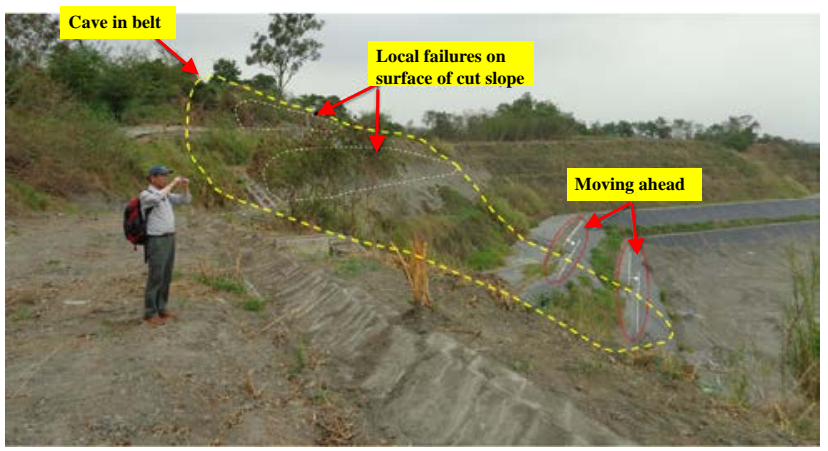

Figure 6. Moving cut slope of Yenchao waste disposal site.

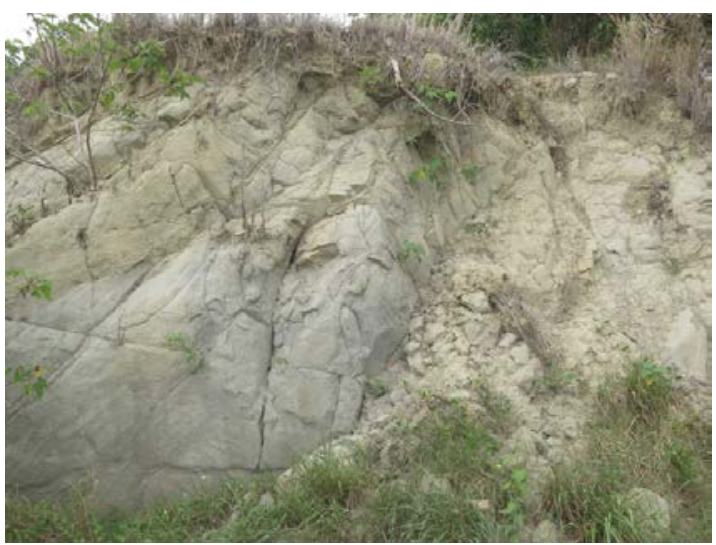

Figure 7. Moving cut slope of Yenchao waste disposal site. 
On late May in 2015, a significant rainfall intensity came out after a long period of dry season which caused water rationing in Kaohsiung as the first time in the history. The rainfall event caused a signal of tilting. The main slope stability, on the other hand, is still being undisturbed during this rain.

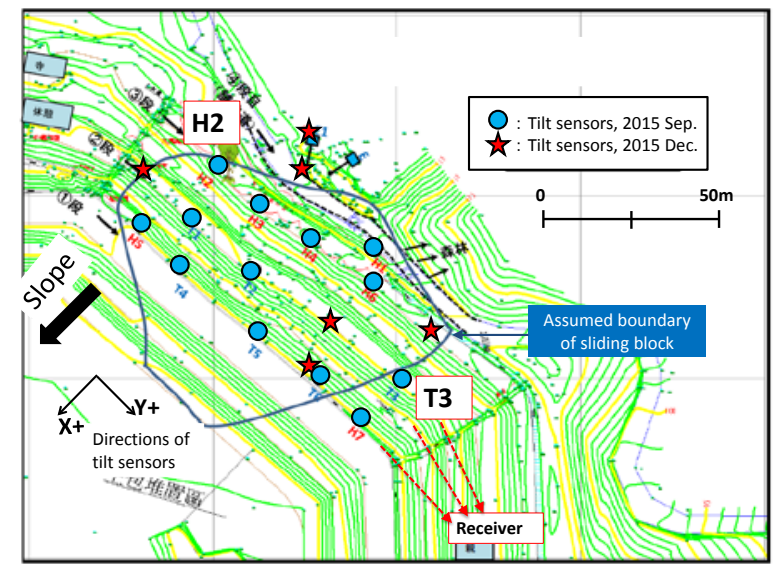

Figure 8. Arrangement of the tilt sensors on the moving cut slope.
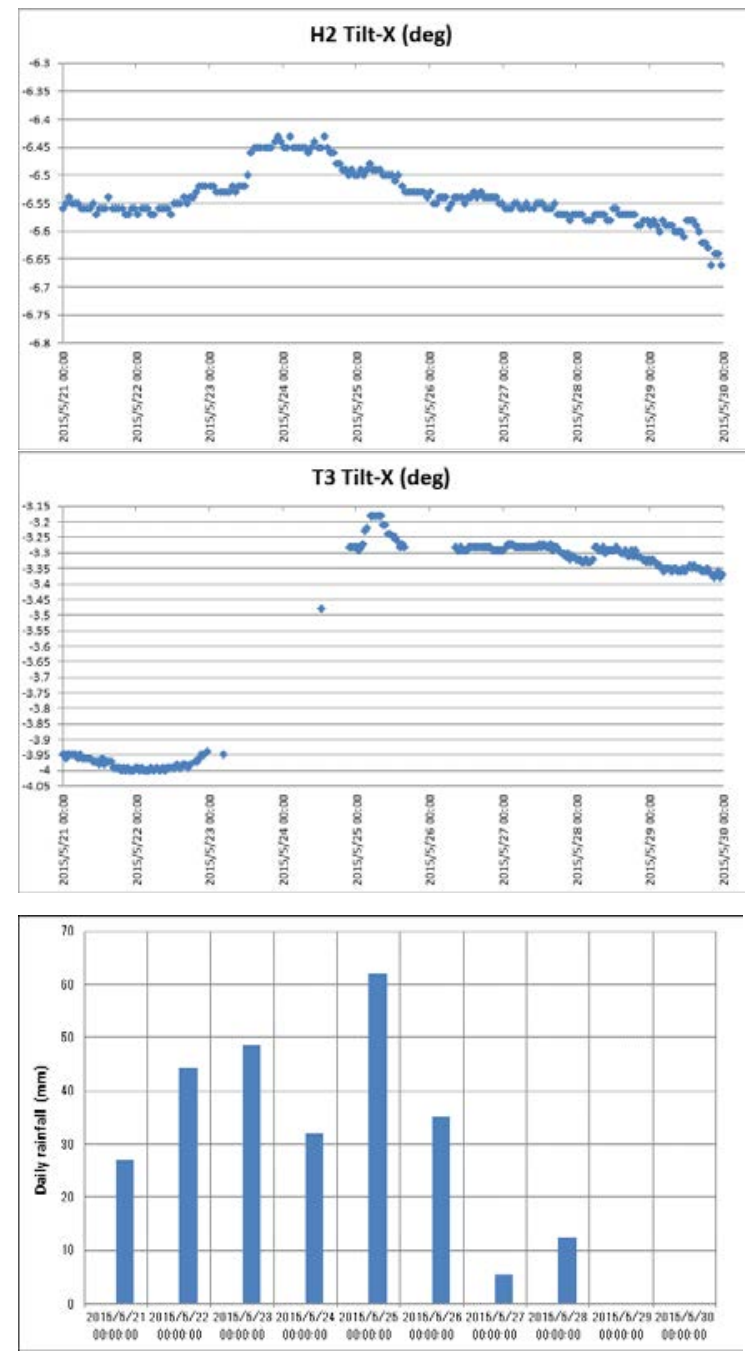

Figure 9. Example of tilt behaviors obtained on the cut slope.
Figure 9 shows a typical tilt behaviors obtained from May 21 to 30 of 2015. The data at H2 and T3 in Figure 8 are indicated for example. The tilt behavior varied by place, and there is some time delay in it after the heavy rainfall event. It is also found that T3, which is out of the assumed sliding block, is also moving by a heavy rainfall event. Distribution of surface tilt behaviors together with their time histories can be analyzed based on these data.

\section{CONCLUSIONS}

A low-cost and simple monitoring method for early warning of rainfall-induced landslides has been proposed. Tilting angles in the surface layer of slope are mainly monitored in this method. In several case studies, it detected distinct behaviors in the tilting angles in the pre-failure stages, and the authors proposed to issue precaution at a tilting rate of 0.01 degrees per hour, and warning at that of 0.1 degree per hour.

The authors attempt to improve the applicability of the moniroting and early warning system by modifying the equipment to be more low-cost, smaller in its size and weight, and simpler. As a result, the total cost for the monitoring sytem is reduced, and larger number of sensors can be installed on the slope site. It helps us to figure out real-time situation of the slope in more details.

The developed prototype of the proposed new sensor units were installed on a cut slope site in Yenchao, Kaohsiung City, Taiwan at the end of 2014. Monitoring has just started, and some tilt behaviors were observed, and more significant behaviors may be observed in the coming rainy season in 2015. The authors will proceed on it, and try to analyze the data to figure out the movement of the sliding block.

\section{ACKNOWLEDGEMENTS}

This research is are supported by SIP (Crossministerial Strategic Innovation Promotion Program) of Cabinet Office of Government of Japan, Grants-in-Aid for Scientific Research of Japan Society for the Promotion of Science (JSPS), and Core-to-Core Program “B. Asia-Africa Science Platforms” (JSPS).

\section{REFERENCES}

1) Uchimura, T., Towhata, I., Trinh, T. L. A., Fukuda, J., Bautista, C. J. B., Wang, L., Seko, I., Uchida, T., Matsuoka, A., Ito, Y., Onda, Y., Iwagami, S., Kim, M. S., and Sakai, N. (2010): "Simple monitoring method for precaution of landslides watching tilting and water contents on slopes surface”, Landslides, (Published online: 17 October 2009)

2) Uchimura, T., Wang, L., Qiao, J.-P., and Towhata, I. (2011). Miniature ground inclinometer for slope monitoring, Proc. of The 14th Asian Regional Conference on Soil Mechanics and Geotechnical Engineering, ATC3 Session.

3) Uchimura, T., Towhata. I., Wang, L., Nishie S., Yamaguchi, H., Seko, I. and Qiao, J.-P. (2015): Precaution and early warning of surface failure of slopes by using tilt sensors, Soils and Foundations, Vol.55, No.5 (to appear). 